associated with different themes of data, with a special focus on health data.

Results There were 11 responders, five parents/guardians and six CYP. Both the parents and the CYP considered personal data, such as date of birth, NI number and name, both the most important and the most private. Health data was considered the second most important, and private, although parents rated data from social media data an equal second in terms of privacy. Within healthcare data, CYP considered data regarding their mental health, followed by medical conditions and genomic data, as the sources to be of highest importance. Parents considered their child's illnesses most important, followed by genomic data. In relation to privacy, CYP considered genomic data first followed by information concerning their mental health. The parents considered genomic data highest for data privacy.

Conclusion From this session it is clear that health data in general, and genetic data in particular, has a high value of importance to CYP and parents, but there are variations in how data is prioritised. These pilot data will inform a large scale patient and parent acceptability study in personalised medicine and childhood asthma (CHANGE study).

Disclosure(s) Nothing to disclose

\section{P46 SPONTANEOUS REPORTING OF SUSPECTED ADVERSE DRUG REACTIONS BY CHILDREN AND YOUNG PEOPLE: PAST, PRESENT AND FUTURE}

\begin{abstract}
${ }^{1} \mathrm{C}$ King, ${ }^{1} \mathrm{~N}$ Bhoombla, ${ }^{1} \mathrm{~J}$ Clarke, ${ }^{1} \mathrm{~V}$ Morgan, ${ }^{2,3}$ J Preston, ${ }^{3} \mathrm{~J}$ Ainsworth, ${ }^{4,5} \mathrm{~A}$ Hanson, ${ }^{2,3} \mathrm{D}$ Hawcutt*. ${ }^{1}$ School of Medicine, University of Liverpool; ${ }^{2}$ Department of Women's and Children's Health, Institute of Translational Medicine, University of Liverpool; ${ }^{3}$ NIHR Alder Hey Clinical Research Facility, Alder Hey Children's NHS Foundation Trust; ${ }^{4}$ Department of Molecular and Clinical Pharmacology, Institute of Translational Medicine, University of Liverpool; ${ }^{5}$ The Royal Liverpool and Broadgreen University Hospitals NHS Trusts, Liverpool, UK
\end{abstract}

\subsection{6/archdischild-2019-esdppp.84}

Background UK patients of all ages can contribute to the Medicines and Healthcare Regulatory Agency (MHRA) Yellow Card scheme (YCS), reporting suspected adverse drug reactions (ADRs). Children's and young people's (CYPs) contribution to, and awareness of, the YCS is not known.

Methods Suspected ADR reports received by the MHRA from 01/01/2008 to 29/11/2018 were examined. Prospective data from CYP and parents in paediatric outpatients were collected as part of the QUestionnaire study about Adverse Drug ReAcTions In Children (QUADRATIC) study (IRAS 242352).

Results CYP contributed 2.3\% of YCS reports for patients < 19 years. Patients from age 10 years old have contributed YCS reports, and CYP reports are increasing annually. Reports from CYP contain different suspected medications and reactions compared to YCS for all patients age $<19$ years. Common CYP generated reports prioritiseadolescent vaccinations (Human papilloma virus most reported, $n=69$ ), oral contraceptives, acne medication, anti-infectives, and antidepressants. Common CYP generated reports identified depression, anxiety and suicidal ideation, which were not amongst common reports about CYP. Amongst CYP $(n=50)$ attending outpatients, the YCS was not known to any CYP. The YCS was known to $16.3 \%$ of parents (8/49) interviewed. From this population of patients with a chronic illness, with high rates of drug therapy, 68 suspected ADRs were identified overall. Conclusion CYP contribute to YCS, with distinct patterns of suspected medications and reactions. Awareness of the YCS appears poor, particularly in populations you would expect to have a greater level of knowledge and understanding about medicines.

Disclosure(s) Nothing to disclose

\section{P47 PREVALENCE, INCIDENCE AND PATTERNS OF ANTIASTHMA MEDICATION USE IN CHILDREN: A NATIONWIDE PRESCRIPTION STUDY IN FRANCE}

${ }^{1}$ I Naim, ${ }^{2} \mathrm{~F}$ Tubach, ${ }^{2} \mathrm{~S}$ Guillo, ${ }^{2} \mathrm{~A}$ Ajrouche, ${ }^{2} \mathrm{Y}$ De Rycke, ${ }^{3} \mathrm{~F}$ Kaguelidou*. ${ }^{1}$ Department of Pediatric Pneumology, Hôpital Robert Debré, APHP, Paris, ${ }^{2}$ Sorbonne Université, INSERM, Institut Pierre Louis d'Épidémiologie et de Santé Publique, IPLESP UMR-S1136, CIC 1421, AP-HP, Hôpital Pitié-Salpêtrière, Département Biostatistique Santé Publique et Information Médicale, Centre de Pharmacoépidémiologie, PARIS; ${ }^{3}$ Department of Pediatric Pharmacology and Pharmacogenetics, Hôpital Robert Debré, APHP; Université Paris VII Diderot - ECEVE - Pres Sorbonne Paris Cité; INSERM CIC1426, APHP - University Paris 7, Paris, France

\subsection{6/archdischild-2019-esdppp.85}

Background Use of antiasthmatic medications has increased over the years worldwide. The aim of this study was to describe the use of antiasthmatic drugs in children in France.

Methods Data were retrieved from the permanent sample (1/ $97 \mathrm{th})$ of the French national healthcare database - the Système National des Données de Santé (SNDS) for all individuals aged from 5 to 18 years old $(n=143,909)$ from 1 January 2011 to 31 December 2017. Prevalence and incidence rate of antiasthmatic dispensing were calculated. All analyses were stratified by calendar year, age (5-11, 12-18 years) and gender. Users were classified as occasional if they had only one dispensing of antiasthmatic drugs over the year, moderate and high if they had antiasthmatic drug dispensing at two distinct occasions or at three or more occasions, respectively.

Results The annual prevalence of antiasthmatic drug use varied between 12 (2011) and 11 (2017) per 100 persons and incidence varied between 4.3 (2013) and 3.8 (2017) per 100 PYs. Prevalence and incidence of use were higher in children aged between 5-11 years compared to adolescents and in boys compared to girls. Most users were occasional (52\%) and only one third redeemed prescriptions on a regular basis (high users: 30\%). No trend was observed regarding these percentages over time or with gender and age group.

Conclusions Use of antiasthmatic drugs in France is higher than previously described in other European countries. Prevalence of use is also higher than the prevalence of asthma as assessed in epidemiological national studies suggesting that these drugs are over-prescribed.

Disclosure(s) Nothing to disclose 